\title{
EgRBP42 from oil palm enhances adaptation to stress tolerance in Arabidopsis through regulation of nucleocytoplasmic transport of stress-responsive mRNAs
}

\begin{abstract}
Abiotic stress reduces plant growth and crop productivity. However, the mechanism underlying posttranscriptional regulations of stress response remains elusive. Herein, we report the posttranscriptional mechanism of nucleocytoplasmic RNA transport of stress-responsive transcripts mediated by EgRBP42, a heterogeneous nuclear ribonucleoprotein-like RNAbinding protein from oil palm, which could be necessary for rapid protein translation to confer abiotic stress tolerance in plants. Transgenic Arabidopsis overexpressing EgRBP42 showed early flowering through alteration of gene expression of flowering regulators and exhibited tolerance towards heat, cold, drought, flood, and salinity stresses with enhanced poststress recovery response by increasing the expression of its target stress-responsive genes. EgRBP42 harbours nucleocytoplasmic shuttling activity mediated by the nuclear localization signal and the M9-like domain of EgRBP42 and interacts directly with regulators in the nucleus, membrane, and the cytoplasm. EgRBP42 regulates the nucleocytoplasmic RNA transport of target stress-responsive transcripts through direct binding to their AG-rich motifs. Additionally, EgRBP42 transcript and protein induction by environmental stimuli are regulated at the transcriptional and posttranscriptional levels. Taken together, the posttranscriptional regulation of RNA transport mediated by EgRBP42 may change the stress-responsive protein profiles under abiotic stress conditions leading to a better adaptation of plants to environmental changes.
\end{abstract}

Keyword: RNA transport; RNA-binding protein; Abiotic stress; Posttranscriptional regulation 